\title{
The Influence of Prior Abdominal Surgery on Robot-Assisted Partial Nephrectomy
}

\author{
Tetsuya Yumioka,* Masashi Honda,* Shogo Teraoka,* Yusuke Kimura,* Hideto Iwamoto,* Shuichi Morizane,* \\ Katsuya Hikita* and Atsushi Takenaka* \\ *Division of Urology, Department of Surgery, School of Medicine, Faculty of Medicine, Tottori University, Yonago 683-8504, Japan
}

\section{ABSTRACT}

Background We evaluated the influence of prior abdominal surgery on perioperative outcomes in patients who underwent robot-assisted partial nephrectomy in initial Japanese series.

Methods We reviewed patients with small renal tumors who underwent robot-assisted partial nephrectomy from October 2011 to September 2020 at our institution. Patients with prior abdominal surgery were compared with those without prior surgery based on perioperative outcomes. The chi-square test and Mann-Whitney $U$ test were used for statistical analyses of variables.

Results Of 156 patients who underwent robot-assisted partial nephrectomy, 90 (58\%) had no prior abdominal surgery, whereas 66 patients (42\%) underwent prior abdominal surgery. No significant differences in perioperative outcomes were observed between with and without prior abdominal surgery groups. In transperitoneal approach robot-assisted partial nephrectomy, 31 patients (80.4\%) had prior abdominal surgery. Trocar insertion time in the with prior abdominal surgery group took longer than the without prior abdominal surgery group (32 vs. $28.5 \mathrm{~min}, P=0.031$ ). No significant difference was observed in the conversion rate between the two groups $(P=0.556)$.

Conclusion Robot-assisted partial nephrectomy appears to be a safe approach for patients with prior abdominal surgery. In transperitoneal approach robotassisted partial nephrectomy with prior abdominal surgery, trocar insertion time was longer, but no significant differences were found in other outcomes. Transperitoneal approach robot-assisted partial nephrectomy is thus considered a safe procedure for patients with prior abdominal surgery.

Corresponding author: Masashi Honda, MD, PhD

honda@tottori-u.ac.jp

Received 2021 March 25

Accepted 2021 April 14

Online published 2021 May 10

Abbreviations: BMI, body mass index; CCI, charlson comorbidity index; EBL, estimated blood loss; eGFR, estimated glomerular filtration rate; LPN, laparoscopic partial nephrectomy; RARP, robot-assisted radical prostatectomy; RAPN, robot-assisted partial nephrectomy; RENAL, radius, exophytic/endophytic properties, nearness to renal sinus, anterior/posterior, and location relative to renal poles; PSMs, positive surgical margins; WIT, warm ischemia time
Key words renal tumor; robotic surgery; partial nephrectomy; prior abdominal surgery; perioperative outcomes

Partial nephrectomy is a gold standard treatment for small renal tumors in patients fit for surgery. ${ }^{1,2}$ Good outcomes have already been proven for both the open and laparoscopic surgery for renal tumors suitable for nephron-sparing surgery. ${ }^{3}$ While a study has demonstrated no significant differences in long-terms oncological outcomes between partial nephrectomy and radical nephrectomy, ${ }^{4}$ partial nephrectomy was superior to radical nephrectomy with respect to length and quality of life..$^{5}$ Laparoscopic partial nephrectomy (LPN) is technically challenging, requiring advanced laparoscopic skills for tumor excision and intracorporeal sutured reconstruction. Following the first report of robot-assisted partial nephrectomy (RAPN) by Gettman et al. in $2004,{ }^{6}$ RAPN has emerged as an alternative to LPN that may help with some of these technical challenges. It has already been demonstrated that RAPN is noninferior to laparoscopic and open approaches with regard to warm ischemia time (WIT), positive surgical margins (PSMs), and perioperative complications. ${ }^{7-10}$ In Japan, RAPN was administered by the Japanese Ministry of Health, Labor and Welfare in 2016. RAPN has become rapidly and widely used in Japan.

Prior abdominal surgery is known to promote adhesion formation; therefore, patients with a history of abdominal surgery have an increased risk of organ injury. Up to $90 \%$ of patients who have undergone open abdominal surgery have been reported to develop peritoneal adhesions. ${ }^{11}$ These adhesions may increase the risk of trocar injuries and perioperative complications and prolong operative time in subsequent procedures. ${ }^{12-15}$ RAPN outcomes after prior abdominal surgery have been previously reported in a few studies. ${ }^{16-18}$

In this study, we evaluated the perioperative outcomes of RAPN in Japanese patients who had undergone prior abdominal surgery. 


\section{MATERIALS AND METHODS \\ Patients}

We retrospectively reviewed the medical records of patients with small renal tumors who underwent RAPN from October 2011 to September 2020 with approval by the ethics committee of Tottori University Faculty of Medicine approved (approval number: 20A165). A history of prior abdominal surgery was confirmed from a medical questionnaire administered at the initial assessment. Perioperative data included surgery duration, estimated blood loss (EBL), number of blood transfusions, WIT, and postoperative complications. RENAL (radius, exophytic/endophytic properties, nearness to renal sinus, anterior/posterior, and location relative to renal poles) nephrometry scores were determined using the formula by Kutikov and Uzzo. ${ }^{19}$ Surgical duration was documented using operating room data sheets, which included total operative time, trocar insertion time, robotic console time, and last incision closure time, as recorded by the operating room nurse. Trocar insertion time was defined as the time from the first incision until the time the robotic system was rolled in. Console time was defined as the time spent by the surgeon using the robotic console. Perioperative complications were classified as grade II or higher using the Clavien-Dindo grading system ${ }^{20}$ Trifecta achieved was defined as no perioperative complications, no PSMs, and WIT $<25$ $\min$.

\section{Surgical technique}

At our institution, the transperitoneal approach involves the standard placement of a 5-trocar system, whereas the retroperitoneal approach entails the standard placement of a 4-trocar system. Camera trocars were placed by open laparotomy. We selected the type of surgical approach for tumor location basically. Adhesiolysis was performed with laparoscopic scissors, as necessary, to allow for the placement of additional trocars and was performed to a considerable extent to enable the placement of the robotic arms and facilitate further lysis of adhesions under robotic assistance.

\section{Statistical analyses}

The patients were divided into two groups: with prior abdominal surgery group and without prior abdominal surgery group. Prior abdominal surgery was defined as laparoscopic and open surgery, which included appendectomy, gynecological surgery, cholecystectomy, hernia repair, colorectomy, gastrectomy, panperitontis, cesarean section, total nephrectomy, robot-assisted radical prostatectomy (RARP), esophagectomy, splenectomy, hepatectomy, and mesenteric tumor resection open biopsy.

The data were analyzed using SPSS v. 21.0 statistical software (IBM, Chicago, IL). Comparison of the medians between groups was made using the MannWhitney $U$ test. The nominal variables were compared using chi-square test. A $P$ value of $<0.05$ was considered to be statistically significant.

\section{RESULTS}

Between October 2011 and September 2020, a total of 156 patients underwent RAPN at our institution. All had complete records of surgical histories, which were included for analysis.

Of the 156 patients, $66(42 \%)$ had a history of abdominal surgery and 90 (58\%) did not. Detailed patient characteristics are listed in Table 1. Significant differences were observed between the two groups in the baseline patient characteristics of age, sex, and ageadjusted Charlson Comorbidity Index (CCI). No significant differences were found among the baseline patient characteristics of body mass index (BMI), tumor size, preoperative estimated glomerular filtration rate (eGFR), RENAL nephrometry score, and type of surgical approach. Table 2 shows the types of abdominal procedures undergone. Fifty-two patients underwent only one prior abdominal surgery, whereas 14 patients underwent multiple abdominal surgeries. Appendectomy was the most common prior surgery $(n=29)$, followed by gynecological surgery $(n=14)$.

Among the 80 patients that underwent transperitoneal RAPN, 31 (38.8\%) had prior history of abdominal surgery and $49(61.2 \%)$ did not. Twenty-six patients had only one prior abdominal surgery, while 5 had undergone multiple prior abdominal surgeries, and appendectomy was the most common prior surgery ( $n$ $=15)$, followed by gynecological surgery $(n=6)$ (Table 2). Table 3 lists baseline patient characteristics and perioperative outcomes for transperitoneal RAPN in patients with prior abdominal surgery compared with patients without prior surgery. Statistically significant differences were observed in age and age-adjusted CCI, but none observed in sex, BMI, tumor size, preoperative eGFR, and RENAL nephrometry scores. For the groups with and without prior surgery, respectively, the median total operative times were 269 and $241 \min (P=0.185)$, the median console times were 182 and $176 \min (P=$ $0.946)$, the median trocar insertion times were 32 and $28.5 \mathrm{~min}(P=0.031)$, median EBL were 25 and $35 \mathrm{~mL}(P$ $=0.373)$, median WIT were 19 and $19 \min (P=0.797)$, conversion rates were $6.5 \%$ and $2.0 \%(P=0.556), \%$ change eGFR were $10 \%$ and $12 \%(P=0.996)$, PSMs were observed in $1(3.4 \%)$ and $0(0 \%)$ patients $(P=0.377)$, 
Table 1. Patients characteristics

\begin{tabular}{|c|c|c|c|c|}
\hline Variable & Total & Prior surgery & No prior surgery & $P$-value \\
\hline Number of patients (\%) & 156 & $\begin{array}{c}66 \\
(42)\end{array}$ & $\begin{array}{c}90 \\
(58)\end{array}$ & \\
\hline Sex (male/female) & $109 / 47$ & $39 / 27$ & $70 / 20$ & 0.014 \\
\hline Age (years; median, IQR) & $\begin{array}{c}65 \\
(34-87)\end{array}$ & $\begin{array}{c}69.5 \\
(43-87)\end{array}$ & $\begin{array}{c}62 \\
(34-86)\end{array}$ & 0.001 \\
\hline Body Mass Index $\left(\mathrm{kg} / \mathrm{m}^{2} ;\right.$ median, IQR) & $\begin{array}{c}23.5 \\
(16.9-42.0)\end{array}$ & $\begin{array}{c}23.0 \\
(18.8-42.0)\end{array}$ & $\begin{array}{c}23.6 \\
(16.9-39.5)\end{array}$ & 0.177 \\
\hline $\begin{array}{l}\text { Age-adjusted Charlson Comobidity Index } \\
\text { (median, IQR) }\end{array}$ & $\begin{array}{c}3 \\
(0-9)\end{array}$ & $\begin{array}{c}4 \\
(0-9)\end{array}$ & $\begin{array}{c}2 \\
(0-6)\end{array}$ & 0.001 \\
\hline Tumor size (mm; median, IQR) & $\begin{array}{c}25 \\
(11-46)\end{array}$ & $\begin{array}{c}25 \\
(12-43)\end{array}$ & $\begin{array}{c}24 \\
(11-46)\end{array}$ & 0.255 \\
\hline Preoperative GFR (mL/min $/ 1.73 \mathrm{~m}^{2} ;$ median, IQR) & $\begin{array}{c}73.2 \\
(17.9-121.3)\end{array}$ & $\begin{array}{c}71.4 \\
(28.5-110.3)\end{array}$ & $\begin{array}{c}74.4 \\
(17.9-121.3)\end{array}$ & 0.305 \\
\hline R.E.N.A.L nephrometry score (median, IQR) & $\begin{array}{c}7 \\
(4-10)\end{array}$ & $\begin{array}{c}7 \\
(4-10)\end{array}$ & $\begin{array}{c}7 \\
(4-10)\end{array}$ & 0.476 \\
\hline Approach (n. Trans/Retro) & $80 / 76$ & $31 / 35$ & $49 / 41$ & 0.418 \\
\hline
\end{tabular}

$\mathrm{IQR}$, inter-quartile range.

Table 2. Type of prior abdominal surgery underwent before RAPN

\begin{tabular}{|c|c|c|c|}
\hline & Total & Transperitoneal & Retroperitoneal \\
\hline Appendectomy & 29 & 15 & 14 \\
\hline Gynecological surgery & 14 & 6 & 8 \\
\hline Cholecystectomy & 8 & 1 & 7 \\
\hline Hernia repair & 6 & 2 & 4 \\
\hline Colorectomy & 6 & 1 & 5 \\
\hline Gastrectomy & 4 & 1 & 3 \\
\hline Panperitontis & 4 & 4 & \\
\hline Caesarean section & 4 & 3 & 1 \\
\hline Radical nephrectomy & 2 & & 2 \\
\hline Robot-assisted radical prostatectomy & 1 & 1 & \\
\hline Open biopsy & 1 & 1 & \\
\hline Esophagectomy & 1 & & 1 \\
\hline Mesenteric tumor resection & 1 & 1 & \\
\hline Splenectomy & 1 & & 1 \\
\hline Hepatectomy & 1 & & 1 \\
\hline Multiple abdominal surgeries & 14 & 5 & 9 \\
\hline
\end{tabular}

blood transfusions were performed in $1(3.4 \%)$ and 0 $(0 \%)$ patients $(P=0.377)$, perioperative complications involved $3(10.3 \%)$ and $5(10.4 \%)$ patients $(P=1.00)$ and trifecta was achieved in $16(55.2 \%)$ and $28(58.3 \%)$ patients $(P=0.816)$.

Among the 76 patients that underwent retroperitoneal RAPN, 35 (46.1\%) had prior history of abdominal surgery and $41(53.9 \%)$ did not. Twenty-six patients had only one prior abdominal surgery, while 9 had undergone multiple prior abdominal surgeries. Table 4 lists baseline patient characteristics and perioperative outcomes for retroperitoneal RAPN in patients with prior abdominal surgery compared with patients without prior surgery. Statistically significant differences were observed in age, sex and age-adjusted CCI, but none observed in BMI, tumor size, preoperative eGFR, 
Table 3. Patients characteristics and preoperative outcomes for Transperitoneal approach

\begin{tabular}{|c|c|c|c|}
\hline Variable & Prior surgery & No prior surgery & $P$-value \\
\hline Number of patients & $\begin{array}{c}31 \\
(38.8)\end{array}$ & $\begin{array}{c}49 \\
(61.2)\end{array}$ & \\
\hline Sex ( $n$. male/female) & $19 / 12$ & $37 / 12$ & 0.214 \\
\hline Age (years; median, IQR) & $\begin{array}{c}67 \\
(43-82)\end{array}$ & $\begin{array}{c}59 \\
(38-84)\end{array}$ & 0.007 \\
\hline Body Mass Index ( $\mathrm{kg} / \mathrm{m}^{2} ;$ median, IQR) & $\begin{array}{c}22.67 \\
(18.8-42)\end{array}$ & $\begin{array}{c}23.70 \\
(16.9-39.5)\end{array}$ & 0.211 \\
\hline Age-adjusted Charlson Comobidity Index (median, IQR) & $\begin{array}{c}3 \\
(0-9)\end{array}$ & $\begin{array}{c}2 \\
(0-3)\end{array}$ & 0.007 \\
\hline Tumor size (mm; median, IQR) & $\begin{array}{c}25 \\
(12-43)\end{array}$ & $\begin{array}{c}26 \\
(15-46)\end{array}$ & 0.382 \\
\hline Preoperative GFR (mL/min $/ 1.73 \mathrm{~m}^{2} ;$ median, IQR) & $\begin{array}{c}77.0 \\
(28.5-104.2)\end{array}$ & $\begin{array}{c}76.4 \\
(24.75-99.88)\end{array}$ & 0.781 \\
\hline R.E.N.A.L nephrometry score (median, IQR) & $\begin{array}{c}7 \\
(4-10)\end{array}$ & $\begin{array}{c}7 \\
(4-10)\end{array}$ & 0.286 \\
\hline Operative time (min; median, IQR) & $\begin{array}{c}269 \\
(177-414)\end{array}$ & $\begin{array}{c}241 \\
(174-46)\end{array}$ & 0.185 \\
\hline Console time (min; median, IQR) & $\begin{array}{c}182 \\
(106-259)\end{array}$ & $\begin{array}{c}176 \\
(119-325)\end{array}$ & 0.946 \\
\hline Trocar insertion time (min; median, IQR) & $\begin{array}{c}32 \\
(20-69)\end{array}$ & $\begin{array}{c}28.5 \\
(12-60)\end{array}$ & 0.031 \\
\hline Estimated blood loss (mL; median, IQR) & $\begin{array}{c}25 \\
(5-400)\end{array}$ & $\begin{array}{c}35 \\
(5-300)\end{array}$ & 0.373 \\
\hline Warm ischemia time (min; median, IQR) & $\begin{array}{c}19 \\
(13-53)\end{array}$ & $\begin{array}{c}19 \\
(15-47)\end{array}$ & 0.797 \\
\hline Conversion $(n . \%)$ & $\begin{array}{c}2 \\
(6.5)\end{array}$ & $\begin{array}{c}1 \\
(2.0)\end{array}$ & 0.556 \\
\hline$\%$ change eGFR $(n . \%)$ & $\begin{array}{c}10 \\
(0-37)\end{array}$ & $\begin{array}{c}12 \\
(0-37)\end{array}$ & 0.996 \\
\hline Positive surgical margin $(n . \%)$ & $\begin{array}{c}1 \\
(3.4)\end{array}$ & $\begin{array}{c}0 \\
(0)\end{array}$ & 0.377 \\
\hline Blood transfusion $(n . \%)$ & $\begin{array}{c}1 \\
(3.4)\end{array}$ & $\begin{array}{c}0 \\
(0)\end{array}$ & 0.377 \\
\hline Perioperative complication Clavien-Dindo $\geq$ II (n.\%) & $\begin{array}{c}3 \\
(10.3)\end{array}$ & $\begin{array}{c}5 \\
(10.4)\end{array}$ & 1.000 \\
\hline Trifecta achievement $(n . \%)$ & $\begin{array}{c}16 \\
(55.2)\end{array}$ & $\begin{array}{c}28 \\
(58.3)\end{array}$ & 0.816 \\
\hline
\end{tabular}

$\mathrm{IQR}$, inter-quartile range.

and RENAL nephrometry scores. For the groups with and without prior surgery, respectively, the median total operative times were 218 and $233 \mathrm{~min}(P=0.091)$, the median console times were 140 and $154 \min (P=0.219)$, median EBL were 10 and $12.5 \mathrm{~mL}(P=0.342)$, median WIT were 20 and $22 \min (P=0.120)$, conversion rates were $8.6 \%$ and $2.4 \%(P=0.329), \%$ change eGFR were $8.6 \%$ and $7.5 \%(P=0.996)$, PSMs were observed in 1 $(2.9 \%)$ and $0(0 \%)$ patients $(P=0.451)$, blood transfusions were performed in $0(0 \%)$ and $1(2.4 \%)$ patients
$(P=1.000)$, perioperative complications involved 3 $(8.6 \%)$ and $4(9.8 \%)$ patients $(P=1.00)$ and trifecta was achieved in $23(65.7 \%)$ and $24(58.5 \%)$ patients $(P=$ 0.452).

The reasons for conversion to radical nephrectomy and open partial nephrectomy are shown in Table 5. Six patients were converted to radical nephrectomy because of uncomplete tumor resection, 1 patient was converted to open partial nephrectomy. However, no significant differences were observed between the patients with and 
Table 4. Patients characteristics and preoperative outcomes for Retroperitoneal approach

\begin{tabular}{|c|c|c|c|}
\hline Variable & Prior surgery & No prior surgery & $P$-value \\
\hline Number of patients & $\begin{array}{c}35 \\
(46.1)\end{array}$ & $\begin{array}{c}41 \\
(53.9)\end{array}$ & \\
\hline Sex ( $n$. male/female) & $20 / 15$ & $33 / 8$ & 0.044 \\
\hline Age (years; median, IQR) & $\begin{array}{c}70 \\
(48-87)\end{array}$ & $\begin{array}{c}63 \\
(34-86)\end{array}$ & 0.003 \\
\hline Body Mass Index ( $\mathrm{kg} / \mathrm{m}^{2} ;$ median, IQR) & $\begin{array}{c}23.4 \\
(18.9-31.2)\end{array}$ & $\begin{array}{c}23.6 \\
(18.3-37.0)\end{array}$ & 0.454 \\
\hline Age-adjusted Charlson Comobidity Index (median, IQR) & $\begin{array}{c}4 \\
(0-9)\end{array}$ & $\begin{array}{c}2 \\
(0-6)\end{array}$ & 0.006 \\
\hline Tumor size (mm; median, IQR) & $\begin{array}{c}23 \\
(12-35)\end{array}$ & $\begin{array}{c}23 \\
(11-42)\end{array}$ & 0.778 \\
\hline Preoperative GFR (mL/min $/ 1.73 \mathrm{~m}^{2} ;$ median, IQR) & $\begin{array}{c}64.4 \\
(31.24-110.2)\end{array}$ & $\begin{array}{c}69.4 \\
(17.87-121.3)\end{array}$ & 0.781 \\
\hline R.E.N.A.L nephrometry score (median, IQR) & $\begin{array}{c}7 \\
(4-10)\end{array}$ & $\begin{array}{c}7 \\
(4-10)\end{array}$ & 0.945 \\
\hline Operative time (min; median, IQR) & $\begin{array}{c}218 \\
(112-295)\end{array}$ & $\begin{array}{c}233 \\
(141-298)\end{array}$ & 0.091 \\
\hline Console time (min; median, IQR) & $\begin{array}{c}140 \\
(69-210)\end{array}$ & $\begin{array}{c}154 \\
(69-207)\end{array}$ & 0.219 \\
\hline Estimated Blood Loss (mL; median, IQR) & $\begin{array}{c}10 \\
(5-250)\end{array}$ & $\begin{array}{c}12.5 \\
(5-400)\end{array}$ & 0.342 \\
\hline Warm ischemia time (min; median, IQR) & $\begin{array}{c}20 \\
(7-31)\end{array}$ & $\begin{array}{c}22 \\
(0-36)\end{array}$ & 0.120 \\
\hline Conversion $(n . \%)$ & $\begin{array}{c}3 \\
(8.6)\end{array}$ & $\begin{array}{c}1 \\
(2.4)\end{array}$ & 0.329 \\
\hline$\%$ change eGFR $(n . \%)$ & $\begin{array}{c}8.6 \\
(0-33)\end{array}$ & $\begin{array}{c}7.5 \\
(0-41)\end{array}$ & 0.738 \\
\hline Positive surgical margin $(n . \%)$ & $\begin{array}{c}1 \\
(2.9)\end{array}$ & $\begin{array}{c}0 \\
(0)\end{array}$ & 0.451 \\
\hline Blood transfusion $(n . \%)$ & $\begin{array}{c}0 \\
(0)\end{array}$ & $\begin{array}{c}1 \\
(2.4)\end{array}$ & 1.000 \\
\hline Perioperative complication Clavien-Dindo $\geq$ II ( $n . \%)$ & $\begin{array}{c}3 \\
(8.6)\end{array}$ & $\begin{array}{c}4 \\
(9.8)\end{array}$ & 1.000 \\
\hline Trifecta achievement $(n . \%)$ & $\begin{array}{c}23 \\
(65.7)\end{array}$ & $\begin{array}{c}24 \\
(58.5)\end{array}$ & 0.452 \\
\hline
\end{tabular}

IQR, inter-quartile range.

without prior abdominal surgery groups in all approaches $(P=0.134)$, the transperitoneal $(P=0.556)$, and retroperitoneal $(P=0.329)$. The conversions were unrelated to history of prior abdominal surgery. However, one patient converted to open partial nephrectomy for strong adhesions. The patients had panperitontis, and strong adhesion between colon and kidney. Therefore, we could not perform adhesiolysis, and opted for open surgery.

\section{DISCUSSION}

In the present study, we retrospectively assessed the influence of prior abdominal surgery on RAPN. There were a few studies for the outcomes of RAPN with prior abdominal surgeries. ${ }^{16-18}$ The present studies is the initial report about influence of prior abdominal surgery on RAPN in Japanese patients.

Generally, previous abdominal surgery is associated with the formation of abdominal adhesions. ${ }^{11}$ Such adhesions have been demonstrated to complicate minimally invasive surgery and prolong operative time. ${ }^{11-15}$ Szomstein et al. ${ }^{21}$ reported that approximately one-third 
Table 5. Conversion and the reasons for conversion

\begin{tabular}{lcccc}
\hline & Total & Prior surgery & No prior surgery & $P$-value \\
\hline Conversion & 7 & 5 & 2 & 0.134 \\
Retroperitoneal approach & 4 & 3 & 1 & 0.329 \\
To radical nephrectomy & 4 & 3 & & \\
Reason & & & 1 & \\
Uncomplete tumor resection & 4 & 3 & 1 & 0.556 \\
Transperitoneal approach & 3 & 2 & 1 & 0 \\
To radical nephrectomy & 2 & 1 & & \\
To open partial nephrectomy & 1 & 1 & 1 \\
Reason & & 1 & 0 \\
Uncomplete tumor resection & 2 & 1 & & \\
Strong adhesions & 1 & & & \\
\hline
\end{tabular}

of patients remain adhesion-free, and up to $10 \%$ of those without a history of abdominal surgery are reported to develop bowel adhesions. However, the type or number of prior abdominal surgeries was unrelated to the severity and type of adhesions. ${ }^{22}$

Several studies have investigated the effects of prior abdominal surgeries on laparoscopic and robotic urological surgeries. ${ }^{22,23}$ Seifman et al. ${ }^{23}$ concluded that previous open abdominal operation increased the risk of operative and major complications and prolonged the length of stay. They evaluated 190 patients who underwent laparoscopic upper tract surgery, 76 of whom had prior abdominal surgery. Patients with prior abdominal surgery had an increased risk of perioperative complications and longer length of stay. On the other hand, Parsons et al. ${ }^{22}$ evaluated 700 patients who underwent laparoscopic urological surgery, of which $366(52 \%)$ had prior abdominal surgery, and found no significant difference in EBL, the rate of conversion to open surgery, and perioperative complications rate. They concluded that prior abdominal surgery does not appear to affect adversely the performance of urological laparoscopy. Several reports have investigated the influence of prior abdominal surgery on RARP. ${ }^{24-26}$ Ginzburg et al. and Siddiqui et al. assessed the influence of prior abdominal surgery on RARP and found that prior abdominal surgery was not associated with an increase in overall operating time, robotic console time, positive surgical margin, EBL, and incidence of complications. ${ }^{16,} 24,25$ We also have previously reported on the influence of prior abdominal surgery on RARP. Of the 150 patients who underwent RARP, 94 (63\%) had no prior abdominal surgery. We found a significant difference in trocar insertion time, but none in total operative time, robotic console time, EBL, and perioperative complications between the two groups. ${ }^{26}$ This study found no significant difference in total operative time, robotic console time, EBL, conversion rate, and perioperative complications between prior abdominal surgery between the patients with and without prior abdominal surgery.

A few reports have been published on the outcomes of RAPN with prior abdominal surgeries. ${ }^{16-18}$ Petros et al. ${ }^{16}$ evaluated that 95 patients underwent transperitoneal RAPN with prior abdominal surgery, 54 had no prior abdominal surgery, whereas 41 patients underwent prior abdominal surgery. There were no significant differences of operative time, WIT, length of hospital stay, and EBL, and significant differences in adhesiolysis. They concluded that transperitoneal RAPN is feasible in the setting of prior abdominal surgery. Similarly, Zangar et al. ${ }^{17}$ evaluated 627 patients who underwent RAPN, 321 of whom had prior abdominal surgery. No significant differences were reported in surgical outcome, operative time, WIT, EBL, and perioperative complications rate (overall Clavien-Dindo grade). They also concluded that RAPN can be safely performed in patients with prior abdominal surgery. Furthermore, Abdullah et al. ${ }^{18}$ reports the first large multi-institutional report on perioperative outcomes of RAPN in patients with prior abdominal surgery. They evaluated 683 patients who underwent RAPN, 216 of whom had had prior abdominal surgery. Their study did not show statistically significant differences in operative time and perioperative complications (Clavien-Dindo $\geq$ III) between patients with and without prior abdominal surgery. However, their study showed higher EBL in the with prior abdominal surgery group. They concluded that RAPN was a safe and feasible option in patients with prior abdominal surgery, and, while an increase in EBL was found, it did not translate into an increase 
in transfusion rate, operative time, or complications. Similarly, we found that prior abdominal surgery did not increase operative time, EBL, WIT, complications, and transfusion rate. Our study concluded that RAPN was a safe procedure in patients with prior abdominal surgery.

Our present study is the first report evaluating the influence of prior abdominal surgery in Japanese patients who underwent RAPN. Significant differences in age, sex, and age-adjusted CCI, but no significant differences were observed in the other preoperative characteristics between the with and without prior abdominal surgery groups. These findings on the influence of prior abdominal surgery are consistent with those in most previous reports with respect to total operative time, robotic console time, EBL, WIT, conversion rate, $\%$ change eGFR, positive surgical margin, blood transfusion rate, and trifecta achievement rate. ${ }^{16-18}$ The perioperative complications rate (Clavien-Dindo $\geq \mathrm{II}$ )for RAPN was $9.6 \%,{ }^{8}$ and for RAPN with prior abdominal surgery was $15.5 \% .^{18}$ The complications rate (ClavienDindo $\geq$ III) for RAPN was $1.0 \%,{ }^{8}$ for RAPN with prior abdominal surgery was $3.1-3.9 \% .{ }^{17,} 18$ In our study, the perioperative complications rate (Clavien-Dindo $\geq \mathrm{II}$ ) for all patients and prior abdominal surgery were $10.1 \%$ and $9.8 \%$. There were no significant differences between prior abdominal surgery and no surgery. Furthermore, we evaluated patients who underwent transperitoneal approach RAPN. Similarly, apart from age and ageadjusted CCI, no significant differences were found in preoperative characteristics, in operative time, robotic console time, EBL, WIT, conversion rate, \% change eGFR, positive surgical margin, complications, and blood transfusion rate. However, a significant difference in trocar insertion time was observed (32 and $28.5 \mathrm{~min}$, respectively; $P=0.031$ ). The results of this study are similar to our previous reports on the influence of prior abdominal surgery on surgical outcomes of RARP. ${ }^{26}$ Therefore, RAPN appears to be a safe procedure for patients with prior abdominal surgery.

The present study showed 7 patients were converted to open partial nephrectomy or radical nephrectomy. The reasons of conversion included incomplete tumor resection $(n=6)$, and strong adhesions $(n=1)$. Arora et al. ${ }^{27}$ reported that RAPN was associated with a low rate of conversion, and independent predictors of conversion were BMI and CCI. Tumor factors such as clinical stage, location, or RENAL score were not associated with increased risk of conversion. In our study, the rate of conversion to open partial nephrectomy or radical nephrectomy were not statistically significant $(P=0.134)$ between patients with and without prior abdominal surgery. In transperitoneal RAPN, 3 patients were converted to open partial nephrectomy or radical nephrectomy. The rates of conversion to open partial nephrectomy or radical nephrectomy were not statistically significant $(P=0.556)$ between patients with and without prior abdominal surgery. The rates of conversion were unrelated to prior abdominal surgery, and prior abdominal surgery was not associated with increased risk of conversion.

Some limitations of our study are to be recognized. First, our study included a single-institution analysis with a small number of patients. Therefore, early cases and various prior abdominal surgeries were included, and all prior abdominal surgeries were comprehensively evaluated. Second, this study involved multiple surgeons. However, the surgeons have performed many robotic surgeries, and the surgical techniques were presumably were same.

In conclusion, RAPN appears to be a safe approach for Japanese patients with prior abdominal surgery. Although an increase in trocar insertion time was observed in transperitoneal approach RAPN with prior abdominal surgery, no significant differences were found in all other perioperative outcomes. Transperitoneal approach RAPN is thus considered a safe procedure for Japanese patients with prior abdominal surgery.

The authors declare no conflict of interest.

\section{REFERENCES}

1 Ljungberg B, Bensalah K, Canfield S, Dabestani S, Hofmann $\mathrm{F}$, Hora $\mathrm{M}$, et al. EAU guidelines on renal cell carcinoma: 2014 update. Eur Urol. 2015;67:913-24. DOI: 10.1016/ j.eururo.2015.01.005, PMID: 25616710

2 Campbell SC, Novick AC, Belldegrun A, Blute ML, Chow GK, Derweesh IH, et al.; Practice Guidelines Committee of the American Urological Association. Guideline for management of the clinical T1 renal mass. J Urol. 2009;182:1271-9. DOI: 10.1016/j.juro.2009.07.004, PMID: 19683266

3 Lane BR, Campbell SC, Gill IS. 10-year oncologic outcomes after laparoscopic and open partial nephrectomy. J Urol. 2013;190:44-9. DOI: 10.1016/j.juro.2012.12.102, PMID: 23306087

4 Russo P, Huang W. The medical and oncological rationale for partial nephrectomy for the treatment of $\mathrm{T} 1$ renal cortical tumors. Urol Clin North Am. 2008;35:635-43; vii. PMID: 18992617

5 Huang WC, Levey AS, Serio AM, Snyder M, Vickers AJ, Raj GV, et al. Chronic kidney disease after nephrectomy in patients with renal cortical tumours: a retrospective cohort study. Lancet Oncol. 2006;7:735-40. DOI: 10.1016/S14702045(06)70803-8, PMID: 16945768

6 Gettman MT, Blute ML, Chow GK, Neururer R, Bartsch G, Peschel R. Robotic-assisted laparoscopic partial nephrectomy: technique and initial clinical experience with da Vinci robotic system. Urology. 2004;64:914-8. DOI: 10.1016/ j.urology.2004.06.049, PMID: 15533477 
7 Porpiglia F, Mari A, Bertolo R, Antonelli A, Bianchi G, Fidanza F, et al. Partial Nephrectomy in Clinical Tlb Renal Tumors: Multicenter Comparative Study of Open, Laparoscopic and Robot-assisted Approach (the RECORd Project). Urology. 2016;89:45-53. DOI: 10.1016/j.urology.2015.08.049, PMID: 26743388

8 Minervini A, Vittori G, Antonelli A, Celia A, Crivellaro S, Dente D, et al. Erratum to: Open versus robotic-assisted partial nephrectomy: a multicenter comparison study of perioperative results and complications. World J Urol. 2014;32:295. DOI: 10.1007/s00345-013-1162-8, PMID: 23913095

9 Lee S, Oh J, Hong SK, Lee SE, Byun SS. Open versus robotassisted partial nephrectomy: effect on clinical outcome. J Endourol. 2011;25:1181-5. DOI: 10.1089/end.2010.0670, PMID: 21657825

10 Ficarra V, Minervini A, Antonelli A, Bhayani S, Guazzoni G, Longo N, et al. A multicentre matched-pair analysis comparing robot-assisted versus open partial nephrectomy. BJU Int. 2014;113:936-41. DOI: 10.1111/bju.12570, PMID: 24219227

11 Liakakos T, Thomakos N, Fine PM, Dervenis C, Young RL. Peritoneal adhesions: etiology, pathophysiology, and clinical significance. Recent advances in prevention and management. Dig Surg. 2001;18:260-73. DOI: 10.1159/000050149, PMID: 11528133

12 Bhoyrul S, Vierra MA, Nezhat CR, Krummel TM, Way LW. Trocar injuries in laparoscopic surgeryl 1No competing interests declared. J Am Coll Surg. 2001;192:677-83. DOI: 10.1016/S1072-7515(01)00913-9, PMID: 11400960

13 Binenbaum SJ, Goldfarb MA. Inadvertent enterotomy in minimally invasive abdominal surgery. JSLS. 2006;10:33640. PMID: 17212891

14 Ellis H, Moran BJ, Thompson JN, Parker MC, Wilson MS, Menzies D, et al. Adhesion-related hospital readmissions after abdominal and pelvic surgery: a retrospective cohort study. Lancet. 1999;353:1476-80. DOI: 10.1016/S01406736(98)09337-4, PMID: 10232313

15 van Goor H. Consequences and complications of peritoneal adhesions. Colorectal Dis. 2007;9(suppl 2):25-34. DOI: 10.1111/j.1463-1318.2007.01358.x, PMID: 17824967

16 Petros FG, Patel MN, Kheterpal E, Siddiqui S, Ross J, Bhandari A, et al. Robotic partial nephrectomy in the setting of prior abdominal surgery. BJU Int. 2011;108:413-9. DOI: 10.1111/j.1464-410X.2010.09803.x, PMID: 21176077

17 Zargar H, Isac W, Autorino R, Khalifeh A, Nemer O, Akca $\mathrm{O}$, et al. Robot-assisted laparoscopic partial nephrectomy in patients with previous abdominal surgery: single center experience. Int J Med Robot. 2015;11:389-94. DOI: 10.1002/ rcs.1633, PMID: 25600756
18 Abdullah N, Rahbar H, Barod R, Dalela D, Larson J, Johnson $\mathrm{M}$, et al. Multicentre outcomes of robot-assisted partial nephrectomy after major open abdominal surgery. BJU Int. 2016;118:298-301. DOI: 10.1111/bju.13408, PMID: 27417163

19 Kutikov A, Uzzo RG. The R.E.N.A.L. nephrometry score: a comprehensive standardized system for quantitating renal tumor size, location and depth. J Urol. 2009;182:844-53. DOI: 10.1016/j.juro.2009.05.035, PMID: 19616235

20 Dindo D, Demartines N, Clavien PA. Classification of surgical complications: a new proposal with evaluation in a cohort of 6336 patients and results of a survey. Ann Surg. 2004;240:205-13. DOI: 10.1097/01.sla.0000133083.54934.ae, PMID: 15273542

21 Szomstein S, Menzo EL, Simpfendorfer C, Zundel N, Rosenthal RJ. Laparoscopic lysis of adhesions. World J Surg. 2006;30:535-40. DOI: 10.1007/s00268-005-7778-0, PMID: 16555020

22 Parsons JK, Jarrett TJ, Chow GK, Kavoussi LR. The effect of previous abdominal surgery on urological laparoscopy. J Urol. 2002;168:2387-90. DOI: 10.1016/S0022-5347(05)641511, PMID: 12441923

23 Seifman BD, Dunn RL, Wolf JS Jr. Transperitoneal laparoscopy into the previously operated abdomen: effect on operative time, length of stay and complications. J Urol. 2003;169:36-40. DOI: 10.1016/S0022-5347(05)64029-3, PMID: 12478097

24 Ginzburg S, Hu F, Staff I, Tortora J, Champagne A, Salner A, et al. Does prior abdominal surgery influence outcomes or complications of robotic-assisted laparoscopic radical prostatectomy? Urology. 2010;76:1125-9. DOI: 10.1016/ j.urology.2010.03.039, PMID: 20708782

25 Siddiqui SA, Krane LS, Bhandari A, Patel MN, Rogers $\mathrm{CG}$, Stricker $\mathrm{H}$, et al. The impact of previous inguinal or abdominal surgery on outcomes after robotic radical prostatectomy. Urology. 2010;75:1079-82. DOI: 10.1016/ j.urology.2009.09.004, PMID: 19896178

26 Yumioka T, Iwamoto H, Masago T, Morizane S, Yao A, Honda $\mathrm{M}$, et al. Robot-assisted radical prostatectomy in an initial Japanese series: the impact of prior abdominal surgery on surgical outcomes. Int J Urol. 2015;22:278-82. DOI: 10.1111/iju.12678, PMID: 25422166

27 Arora S, Chun B, Ahlawat RK, Abaza R, Adshead J, Porter JR, et al. Conversion of Robot-assisted Partial Nephrectomy to Radical Nephrectomy: A Prospective Multiinstitutional Study. Urology. 2018;113:85-90. DOI: 10.1016/ j.urology.2017.11.046, PMID: 29284123 